\section{Cahiers de Narratologie}

Analyse et théorie narratives

$10.2 \mid 2001$

La voix narrative

\title{
Symphonie de Salieri. Voix dans Amadeus
}

\section{Shenghui Lu}

\section{OpenEdition}

Journals

Édition électronique

URL : http://journals.openedition.org/narratologie/10215

DOI : 10.4000/narratologie. 10215

ISSN : 1765-307X

Éditeur

LIRCES

Édition imprimée

Date de publication : 1 janvier 2001

Pagination : 193-202

ISBN : 2914561032

ISSN : 0993-8516

\section{Référence électronique}

Shenghui Lu, «Symphonie de Salieri. Voix dans Amadeus », Cahiers de Narratologie [En ligne], 10.2 | 2001, mis en ligne le 01 janvier 2001, consulté le 11 juin 2020. URL : http://journals.openedition.org/ narratologie/10215 ; DOI : https://doi.org/10.4000/narratologie.10215 


\title{
SYMPHONIE DE SALIERI. VOIX DANS AMADEUS
}

\author{
Shenghui LU \\ Université de Nankin (Chine)
}

Amadeus de Milos Forman est un film de grand succès ( 8 Oscars en 1984). Bien des critiques de cinéma ont étudié sa mise en scène, sa musique ainsi que sa véracité historique. Les techniques narratives employées dans ce film méritent pourtant des réflexions méthodologiques approfondies.

En effet, par sa structure rétrospective et par l'ambiguïté de la voix d'Antonio Salieri, ce film sur Wolfgang Amadeus Mozart fournit un bel exemple permettant d'aborder des questions narratologiques. Car, Salieri y joue un triple rôle de narrateur (1), de personnage (2) et d'interprète de l'œuvre de Mozart (3).

\section{Qui est le narrateur?}

La question du narrateur au cinéma s'est posée dès les années 50, notamment avec le Roman d'un tricheur (1936) de S. Guitry, Lady in the Lake (1946) de R. Montgomery et le Journal d'un curé de campagne (1951) de R. Bresson. En théorie littéraire, G. Genette et la plupart des narratologues français fondent leur typologie sur la notion de narrateur considéré comme une présence anthropomorphique ou une instance narrative. Au contraire, selon des néo-formalistes anglo-américains soutenant la thèse du non-narrateur, tels que S. Chatman ${ }^{1}$ et $\mathrm{S}$. Kozloff ${ }^{2}$, la narration filmique est construite sur le modèle d'une énonciation sans personne, c'est-à-dire sans présence humaine ni sujet parlant unifié. Chez Ch. Metz, l'énonciation filmique, de caractère impersonnel, ne désigne

I S. ChATMAN : Story and Discourse, Ithca et Londres, Cornell University Press, 1978.

2 S. KOZLOFF : Invisible Storyteller, Berkeley, Los Angeles et Londres, University of California, 1988. 
que «l'activité qui produit l'énoncé, l'acte filmique comme tel, toujours identique à lui-même en son fond $»^{3}$.

Or, dans Amadeus, le narrateur, divisé et décentralisé, est analysable à plusieurs niveaux.

Le récit premier du film commence par la séquence du suicide de Salieri. Derrière une porte fermée, un homme hurle et implore : "Pardonne, Mozart, pardonne à ton assassin ! " L'histoire de Mozart débute au moment où Salieri, enfermé dans un hôpital de malades mentaux, se confesse à un prêtre. Le récit premier relève d'un mode de narration centrale ; celuici fonctionne avec le dispositif filmique qu'est la caméra omnisciente et omniprésente, dont le présent correspond au passé romanesque. A la fin du film, le récit premier se poursuit ; Salieri continue sa confession après l'enterrement de Mozart, s'adresse, en " champion des médiocres ", à ses pairs : «Je vous absous. Je vous absous. »

Le récit secondaire (sur le plan narratologique) n'est plus l'histoire de Salieri, mais celle d'Amadeus, ou plus exactement l'épisode de la vie de Mozart à Vienne entre 1781 et 1791.

Contrairement à une idée de M. Sineux, cet épisode n'est pas « vu du seul point de vue de Salieri » ${ }^{4}$. Plusieurs scènes ne sont pas racontées par Salieri : par exemple, la démarche faite par Léopold Mozart à Salzbourg avant le mariage de son fils à Vienne, l'assistance de Mozart à une représentation théâtrale de Schkanader, l'arrivée du père de Mozart à Vienne, etc. Théoriquement, il est impossible pour Salieri de les relater ; car un personnage ne peut raconter que des événements dont il est témoin, s'agissant d'un inconvénient typique du narrateur « je ».

L'histoire de Mozart est donc relatée soit directement par le dispositif impersonnel du film, soit à travers le récit de Salieri. Ce double point de vue est affirmé par M. Forman : «la plupart du temps, nous avons envisagé les scènes du point de vue de Salieri ; ensuite, la vie se déroule d'elle-même ; nous n'avons pas eu le projet de juger la vision de Salieri, de la distinguer de ce que nous pensions être l'objectivité, car nous 1991.

3 Ch. METZ : L'Enonciation impersonnelle, Paris, Klincksieck,

4 M. SINEUX : "Quasi una fantasia », Positif, Paris, nov. 1984, $\mathrm{n}^{\circ} 285$, p. 30 . 
ne savons pas nous-même ce qui s'est passé en réalité, ni comment. Il aurait été prétentieux de tenter de faire la différence entre la vision de Salieri et la réalité objective. ${ }^{5} \mathrm{Si}$ cette citation vise à défendre l'unité de la vie de Mozart saisie par le film, elle atteste, en même temps, l'existence d'un double point de vue. De plus, elle ressemble à une affirmation de Benveniste : "A vrai dire, il n'y a même plus alors de narrateur. Les événements sont posés comme ils se sont produits à mesure qu'ils apparaissent à l'horizon de l'histoire. Personne ne parle ici ; les événements semblent se raconter eux-mêmes. Le temps fondamental est l'aoriste qui est le temps de l'événement hors de la personne d'un narrateur. ${ }^{6}$ Il s'agit de l'« histoire » de Benveniste, d'un mode de narration, où le narrateur, omniprésent et omniscient, s'identifie aux personnages.

Toutefois, comme l'a dit M. Forman, le récit secondaire est essentiellement narré par Salieri, à qui le narrateur central délègue son pouvoir narratif. Bien qu'il considère aussi que : « nous voyons Amadeus par les yeux de Salieri, c'est lui qui nous guide, à travers le récit de sa confession, vers Mozart ${ }^{7}$, S. Toubiana a insisté sur la structure panoptique du film.

Un extrait de la séquence, où Constance Mozart présente à Salieri une partition originale de Mozart en vue de son aide, illustre bien ce mode de narration ${ }^{8}$.

5 «Chaque personnage a ses raisons. Entretien avec Milos Forman ", Cahiers du cinéma, Paris, nov. 1984, n³65, p. 10.

6 E. BENVEnISTE : Problèmes de linguistique générale, t. 1, Paris, Gallimard, 1966, p. 241.

7 S. TOUbianA : «Le Regard du sourd », Cahiers du cinéma, op. cit., p. 6.

${ }^{8}$ Les découpages sont faits, à titre indicatif, par l'auteur de cet article pour son analyse du film. Par commodité, sont utilisées les abréviations suivantes : S (Salieri) ; Z (Stanzi, Constance); PR (plan rapproché) ; PM (plan moyen); PE (plan éloigné) ; GP (gros plan) ; C (champ); CC (contre-champ); C-CC (champ-contre-champ). 


\begin{tabular}{|c|c|c|c|}
\hline No. & Figure & $\begin{array}{c}\text { Image } \\
\text { Montage }\end{array}$ & Discours verbal \\
\hline 0 & S lit la partition, questionne $\mathrm{Z}$, dont seul le chapeau est visible. & $\mathrm{PR}, \mathrm{C}$ & $\begin{array}{l}\text { Alors, ce sont des } \\
\text { originaux? }\end{array}$ \\
\hline 1 & En goûtant une friandise, $\mathrm{Z}$ fait signe de tête affirmatif. & $\mathrm{PE}, \mathrm{CC}$ & Mhmm (« Oui $n)$ \\
\hline 2 & La partition. & GP CC & \\
\hline 3 & La partition dans ses mains, $\mathrm{S}$ la lit, se lève et quitte la table. & PR & \\
\hline 4 & En mangeant sa friandise, $\mathrm{Z}$ regarde $\mathrm{S}$. & PR C & \\
\hline 5 & S, de dos, tient la partition dans ses mains. & PM CC & \\
\hline 6 & S regarde la partition. & PR CC & \\
\hline 7 & $\mathrm{Z}$, assise devant la table, prend une autre friandise, en regardant $\mathrm{S}$. & PE C & \\
\hline 8 & Idem 6. & PE C & \\
\hline \multicolumn{4}{|c|}{ (Par commodité pour l'analyse, les plans 9 à 18 de la même séquence sont décrits ci-après.) } \\
\hline 19 & Idem 6 (mais S en extase). & GP C & \\
\hline 20 & La partition tombe des mains de S. & GP CC & \\
\hline 21 & $\mathrm{Z}$ regarde $\mathrm{S}$. & GP & \\
\hline 22 & Les feuilles tombées par terre, aux pieds de $\mathrm{S}$. & PR & \\
\hline 23 & $\mathrm{Z}$ regarde $\mathrm{S}$. & PR C & C'est sans valeur? \\
\hline 24 & S se toume vers $\bar{Z}$. & PR C-CC & C'est miraculeux. \\
\hline 25 & S sourit, se lève et se dirige vers $\mathrm{S}$. & PR C-CC & Oh ! Oui. \\
\hline 26 & $\begin{array}{l}\mathrm{S} \text { regarde } \mathrm{Z} \text {, qui s'approche de lui, ramasse les feuilles, tout en le } \\
\text { regardant. }\end{array}$ & PR C-CC & $\begin{array}{l}\text { Il est très fier de son } \\
\text { travail. }\end{array}$ \\
\hline 27 & $\mathrm{~S}$ regarde $\mathrm{Z}$ & PR C-CC & $\begin{array}{l}\text { Alors, vous nous } \\
\text { aiderez, Monsieur? }\end{array}$ \\
\hline 28 & S donne le cahier à Z, qui l'accepte. & PM & \\
\hline 29 & Idem 27. & & \\
\hline 30 & S se tourne et se dirige vers la porte, ferme la porte ; $\mathrm{Z}$ le regarde. & PM & \\
\hline
\end{tabular}

Tel que ce tableau le décrit, les prises de vue sont généralement des plans rapprochés ou de gros plans susceptibles de montrer les portraits des personnages. L'intensité dramatique de cette séquence autant que du film entier est assurée par le cut et le champ-contre-champ.

Comme Salieri, narrateur, est aussi un personnage du récit secondaire, la narration se déroule à travers la caméra, les dialogues étant au présent. Le film ne recourt pas à des procédés de distanciation suggérés par Brecht ${ }^{9}$ pour rendre possible la distanciation entre spectateur et personnagenarrateur. Le personnage s'identifie au narrateur, qui ne peut plus être omniscient. La voix du personnage de Salieri ici n'est pas une voix off au sens propre du terme. Elle diffère de la voix off dans Le Sorgho rouge de Zhang Yimou, dans Paulina 1880 de J.-L. Bertuccelli ou dans L'Idiot de

9 B. BRECHT : Ecrits sur le théâtre, t. I, Paris, L'Arche, 1972, p. 333. 
Kurosawa10. Il s'agit du récit de paroles, que G. Genette considère comme le seul procédé du showing par opposition au telling ${ }^{11}$.

\section{Qui parle à Dieu?}

L'ambiguïté de la voix de Salieri se trouve non seulement dans ses paroles dites à des personnages du récit secondaire, mais aussi dans sa voix adressée à Dieu. A cet égard, il semble que seul le monologue intérieur soit propre à rendre transparente la vie intérieure de Salieri. Ce mode narratif, faisant partie d'un ensemble de procédés appropriés au courant de conscience, se transforme en mode de langage après Joyce et Faulkner. Comme Paulina chez Jouve, Salieri, enfermé dans un asile, se livre sans cesse au monologue intérieur. Il communique avec Dieu souvent sous forme d'apostrophe.

\begin{tabular}{|l|l|l|l|}
\hline No. & \multicolumn{1}{|c|}{ Figure } & \multicolumn{1}{|c|}{$\begin{array}{c}\text { Image } \\
\text { Montage }\end{array}$} & \multicolumn{1}{|c|}{ Discours verbal } \\
\hline 0 & S parle au prêtre (caché). & GP & Désormais, nous deux, nous sommes deux ennemis, toi et moi, \\
\hline 1 & $\begin{array}{l}\text { Un crucifix fixé au mur ; une } \\
\text { main de S apparaît, le prend. }\end{array}$ & PM & $\begin{array}{l}\text { Parce que tu as choisi comme interprète ce vantard, ce garçon } \\
\text { obscène, grivois, infantile, et que ma seule récompense sera } \\
\text { d'être le seul à reconnaître ton incarnation. Puisque tu es injuste }\end{array}$ \\
\hline 2 & S le met au feu. & PR & Déloyal, cruel, \\
\hline 3 & S regarde la flamme. & PR C & Je te ferai obstacle. Je le jure. Je t'entraverai. \\
\hline 4 & Le feu brûle le crucifix. & PR CC & Je ferai du mal à ta créature sur terre, de toutes mes forces. \\
\hline 5 & Idem 0. & PR & Oui, moi, je vais ruiner ton incarnation. \\
\hline
\end{tabular}

Cette courte séquence suit, par le montage cut, celle où Constance présente à Salieri une partition originale de Mozart. Elle montre un glissement de niveaux de récits et de voix, lequel caractérise Amadeus de M. Forman, comme La Flûte enchantée de Mozart se distingue par une symbiose de modes d'expression.

$10 \mathrm{Cf}$. S. LU : Transformation et réception du texte par le film, (Bern, Peter Lang, 1999), qui propose une nouvelle approche de l'adaptation fondée sur cinq exemples: L'Idiot (Dostoïevski et Kurosawa); Le Sorgho rouge (Mo Yan et Zhang Yimou) ; Journal d'un curé de campagne (Bernanos et Bresson); Paulina 1880 (Jouve et Bertuccelli) ; Macbeth (Shakespeare et Welles).

11 G. GENETTE : Figures III, Paris, Seuil, 1973, pp. 189-203. 
Les plans 0 et 5 , où Salieri se confesse devant le prêtre, se situent au niveau du récit premier. En revanche, les plans 1 à 4 ne peuvent se situer qu'au niveau du récit secondaire, où Salieri agit en tant que personnage. Les raisons sont très simples : il n'y a pas de brasier dans l'asile ; un prêtre ne tolère pas qu'on brûle un crucifix devant lui ; dans les plans 1 à 4 , Salieri est en costume aristocratique (comme dans la séquence précédente), alors qu'il s'habille en malade mental dans les plans 0 et 5 . Néanmoins, les paroles adressées à Dieu, toutes au présent, sont si harmonieuses qu'un décalage se produit entre image et bande-son. Au dernier plan (5) de cette séquence, Salieri, après avoir parlé à Dieu, semble sourire au prêtre toujours invisible, comme s'il s'adressait à un allocutaire. Comme le monologue intérieur est un discours immédiat, Deleuze $^{12}$, après Eisenstein, a insisté sur sa puissance virtuelle au cinéma.

L'identification aristotélicienne s'établit a priori entre salle et scène, spectateur et personnage. Cependant, pour que le spectateur éprouve un certain sentiment de transfert psychologique ou une empathie cathartique, il faut que le narrateur s'assimile aux personnages. Pour que le contact s'établisse entre salle et scène, il faut qu'il s'organise en scène, entre narrateur et personnages. Le monologue intérieur relève d'un mode de représentation identificatoire, surtout lorsqu'il s'agit du monologue intérieur surgissant comme flux de conscience. Au cinéma, ce type d'images d'identification sont souvent qualifiées d'objectives ou de neutres. Ch. Metz en a pourtant insisté sur le caractère relatif : "plus le public est éduqué, plus le nombre d'images neutres diminue $»^{13}$.

\section{Et le spectateur...}

M. Forman met en œuvre une stratégie pragmatique du spectateur, laquelle est réalisée par le choix du personnage du prêtre et par la voix de Salieri commentant l'œuvre de Mozart.

Le prêtre joue un rôle secondaire sur le plan des actions, mais très important d'un double point de vue esthétique et

12 G. DELEUZE : L'Image-Temps : Cinéma 2, Paris, Eds. de Minuit, 1985, pp. 206-208 et 237-238.

13 Ch. METZ : L'Enonciation impersonnelle, op. cit., p. 169. 
pragmatique. Au niveau esthétique, il s'agit d'épouser le réalisme cinématographique : "Le cinéma est beaucoup plus réaliste que le théâtre ; par nature au théâtre, tout est stylisé sur scène. Le cinéma doit être beaucoup plus réaliste. Et s'il faut du réalisme, il est absolument naturel d'introduire par exemple le personnage du prêtre. $»^{14}$

Sur le pragmatique, M. Forman a justifié son choix de façon convaincante : "j'avais des doutes quant à l'utilisation du même 'truc' qu'à la scène. Cela me rappelait trop les émissions de télévision où l'on vous parle en gros plan. Je suis parti de la situation réelle. Un vieil homme a tenté de se suicider. On l'a transporté à l'asile. A cette époque on ne consultait pas un psychiatre mais un prêtre. Cela me semblait convenir parfaitement. Le prêtre bien sûr représenterait plus ou moins les spectateurs. Il serait donc passif. " ${ }^{15} \mathrm{C}^{\prime}$ est à travers le prêtre que Salieri remplit sa fonction de narrateur et raconte l'histoire de Mozart au spectateur. A ce titre, il paraît intéressant de concevoir, comme chez E. Branigan ${ }^{16}$, la narration dans la perspective pragmatique, ou de construire le narrateur "pour mieux conférer au procès de narration le pouvoir de signaler, dans certaines circonstances, que le spectateur devrait construire un narrateur ${ }^{17}$.

Cependant, Salieri est libre devant son prêtre, autant qu'un fou peut l'être devant le sien. Il s'adresse au spectateur souvent à travers un "troisième personnage ", qui est la musique, en particulier la musique de Mozart. M. Forman veut «que la musique soit le troisième personnage de l'histoire et qu'elle soit en rapport avec cette histoire, qu'elle fasse même avancer l'intrigue. Elle ne devait en aucune façon jouer le rôle d'intermède. "18 Dans Amadeus, la musique est rarement off.

14 «Chaque personnage a ses raisons. Entretien avec Milos Forman ", Cahiers du cinéma, op. cit., p. 10.

15 M. CIMENT : «Entretien avec Milos Forman », Positif, op. cit., p. 23.

16 E. BRANIGAN : Point of View in the Cinema, Berlin / New York / Amsterdam, Mouton Publisher, 1984.

17 D. BORDWELL : Narration in the Fiction Film, Madison, University of Wisconsin Press, 1985, p. 62.

18 M. CIMENT : «Entretien avec Milos Forman », Positif, op. cit., p. 25. 
Elle ne joue pas le rôle d'intermède comme au théâtre qui y résiste ; elle n'accompagne pas l'histoire comme dans biens des films ; mais elle s'intègre dans l'histoire et dans la vie des protagonistes. Dès lors, c'est Salieri, compositeur de la Cour, qui la commente. N'est-ce pas une belle leçon de musique de Mozart pour le public?

Exemple. A partir du plan 2 (du tableau I) s'élève une musique sublime devant laquelle s'extasie le rival italien du virtuose précoce de Salzbourg. La musique de Mozart est coupée brusquement, au plan 19, par le bruit des feuilles de notes qui tombent des mains de Salieri.

\begin{tabular}{|c|c|c|c|}
\hline No. & Figure & $\begin{array}{l}\text { Image } \\
\text { Montage }\end{array}$ & Discours verbal \\
\hline \multicolumn{4}{|c|}{ (Les plans 0 à 8 et 18 à 30 de la même séquence sont décrits dans le tableau l.) } \\
\hline 9 & $\begin{array}{l}\text { La partition lue par } \mathrm{S} \text {, } \\
\text { dont on voit la nuque. }\end{array}$ & GP & Stupéfiant. \\
\hline 10 & $\begin{array}{l}\text { S parle au prêtre } \\
\text { caché. }\end{array}$ & PR & Prodigieux. On croyait vivre un rêve. \\
\hline 11 & Idem 6. & PR C & C'était là un premier, seul et unique, premier jet. \\
\hline 12 & La partition. & GPCC & Mais, on n'y voyait aucune correction, d'aucune sorte, pas une seule. \\
\hline 13 & Idem 9. & GP & II n'avait eu qu'à transcrire sur le papier \\
\hline 14 & Idem 10 & PR & $\begin{array}{l}\text { une musique achevée dans sa tête, page après page, comme s'il } \\
\text { l'avait prise sous la dictée. }\end{array}$ \\
\hline 15 & Idem 6. & PR & \\
\hline 16 & Idem 10. & PR & $\begin{array}{l}\text { Une musique achevée comme jamais ma musique ne put l'être. } \\
\text { Déplacer une seule note, on allait vers l'amoindrissement ; déplacer } \\
\text { une seule phrase, on aurait vu la structure s'effondrer. }\end{array}$ \\
\hline 17 & Idem 6 . & PR & $\begin{array}{l}\text { C'était pour moi très clair. Les sonorités que j'avais entendues dans } \\
\text { le salon de l'Archevêque n'avait pas été un accident. Là encore } \\
\text { c'était la voix même de Dieux. }\end{array}$ \\
\hline 18 & Idem 10 ( $\mathrm{S}$ en extase). & GP & $\begin{array}{l}\text { Je scrutai au-delà de la cage d'une partition méticuleusement griffée } \\
\text { de notes, tout un absolu de beauté. }\end{array}$ \\
\hline
\end{tabular}

Comme ce tableau le montre, le commentaire de Salieri est en mode narratif, au passé. Les 10 plans se regroupent presque en deux : dans le plan $10,14,16$ et 18 , Salieri parle au prêtre, qui est le spectateur supposé ; dans le plan 6, 11, 15 et 17, Salieri lit la partition, la voix hors champ (de Salieri devant le prêtre) commentant la musique, comme s'il s'adressait au spectateur. M. Ciment a montré le caractère pédagogique de deux scènes, lorsqu'il interviewait le réalisateur : "La scène où Mozart dicte le Requiem à Salieri et celle où Salieri explique l'adagio de la Sérénade pour instruments à vent sont de merveilleuses dramatisations de l'esthétique musicale et ont en même temps un caractère presque didactique pour le 
public. ${ }^{19}$ A ce titre, l'inscription du spectateur dans le film résulte des « déterminations institutionnelles ${ }^{20}$ du cinéma et se fonde sur des raisons pragmatiques ${ }^{21}$.

Les plus beaux extraits de musique de Mozart sont commentés par Salieri en personnage (la voix n'étant pas off) et narrateur (le temps étant au passé). Il en est ainsi pour L'Enlèvement au sérail et pour Les Noces de Figaro (même après les explications de Mozart pour convaincre l'empereur Joseph II). Sauf La Flûte enchantée. Mais on comprend cette " absence » de Salieri, qui veut saisir une belle occasion de " passer à l'acte de meurtre ». C'est le génie lui-même qui commente son Requiem. Cette séquence, où Mozart, mourant, dicte son requiem à Salieri, éclaire excellemment le processus de création chez Mozart.

Quant à Don Giovanni, Salieri va jusqu'à en fournir une interprétation génétique établissant les rapports entre l'apparition du Commandeur et la mort de Léopold Mozart : "Le soir, j'étais là, comprenant que ce vieillard amer gardait une emprise sur son pauvre fils, bien qu'il fût outre-tombe. J'envisageai une façon, une façon ignoble d'assurer, en fin de honte, mon triomphe sur Dieu. [...] C'est de là que naquit, terrifiant, le spectre de son opéra futur, son plus noir opéra. L'âme sur la scène se donnait l'image du Commandeur mort. Je sus, je fus le seul à deviner que l'horrible apparition était Léopold resurgi de l'empire des morts. " Cette analyse, partagée par le cinéaste, est corroborée par le montage cut; celui-ci, faisant le changement brusque entre l'appartement, où apparaît le portrait, fixé au mur, de Léopold Mozart qui vient de mourir, et la séquence, où surgit le Commandeur faisant irruption sur la scène, en traversant un mur en carton.

En conclusion, le cinéma, par sa forme hétérogène et complexe, est considéré comme un champ d'analyse méthodologique par excellence. Dans Amadeus de M. Forman, la

19 Ibid., p. 24.

20 R. ODIN : «Du spectateur fonctionnalisant au nouveau spectateur ", Iris, Paris, n 8, 1988, pp. 121-139.

21 F. CASETTI : D'un regard l'autre. Le Film et son spectateur, Lyon, Presses universitaires de Lyon, 1990. 
voix de Salieri est une symphonie, où l'on entend à la fois une voix narrative racontant l'histoire, une voix monologique adressée à Dieu et une voix pragmatique destinée au spectateur.

Dans un discours narratif, le narrateur existe, sous une forme ou sous une autre, en qualité de personnage ou à titre d'instance narrative, se montre ou se dissimule. De même que la concordance narrative ne rompt pas totalement avec l'esthétique aristotélicienne, de même l'intervention d'un narrateur entre en rapport avec la distanciation. La conception brechtienne du théâtre épique, privilégiant les relations de distance, garantit le jeu de la distanciation par des techniques spécifiques, comme dans l'opéra de Pékin.

Dès les années 50 , A. Bazin a remis en cause la thèse opposant le théâtre au cinéma et au roman comme on oppose la distanciation à l'identification : "Théâtre et cinéma ne seraient donc plus séparés par un fossé esthétique infranchissable, ils tendraient seulement à susciter deux positions mentales sur lesquelles les metteurs en scène gardent un large contrôle. ${ }^{22}$ Les techniques de la mise en scène dont le cinéma et le roman disposent sont aussi appropriées à susciter la passivité de l'identification qu'à provoquer la conscience critique de la distanciation. Ce n'est pas les supports, mais les modes d'expression et d'affichage du narrateur, qui entraînent l'identification ou la distanciation. Car, les différents sujets du discours artistique entretiennent de multiples rapports fluctuants entre eux.

22 A. BAZIN : "Théâtre et cinéma ", Qu'est-ce que le cinéma?, Paris, Eds. du Cerf, 1987, p. 154. 